\title{
Upper bounds for the number of spanning trees of graphs
}

\section{ફ̧B Burcu Bozkurt*}

\section{"Correspondence:}

sbbozkurt@selcuk.edu.tr

Department of Mathematics,

Science Faculty, Selçuk University,

Campus, Konya, 42075, Turkey

\begin{abstract}
In this paper, we present some upper bounds for the number of spanning trees of graphs in terms of the number of vertices, the number of edges and the vertex degrees.
\end{abstract}

MSC: $05 C 05 ; 05 C 50$

Keywords: graph; spanning trees; normalized Laplacian eigenvalues

\section{Introduction}

Let $G$ be a simple graph with $n$ vertices and $e$ edges. Let $V(G)=\left\{v_{1}, v_{2}, \ldots, v_{n}\right\}$ be the vertex set of $G$. If two vertices $v_{i}$ and $v_{j}$ are adjacent, then we use the notation $v_{i} \sim v_{j}$. For $v_{i} \in V(G)$, the degree of the vertex $v_{i}$, denoted by $d_{i}$, is the number of vertices adjacent to $v_{i}$. Throughout this paper, we assume that the vertex degrees are ordered by $d_{1} \geq d_{2} \geq \cdots \geq d_{n}$.

The complete graph, the complete bipartite graph and the star of order $n$ are denoted by $K_{n}, K_{p, q}(p+q=n)$ and $S_{n}$, respectively. Let $G-m$ be the graph obtained by deleting any edge $m$ from the graph $G$ and let $\bar{G}$ be the complement of $G$. Let $G \cup H$ be the vertexdisjoint union of the graphs $G$ and $H$ and let $G \vee H$ be the graph obtained from $G \cup H$ by adding all possible edges from vertices of $G$ to vertices of $H$, i.e., $G \vee H=\overline{\bar{G} \cup \bar{H}}$ [1].

Let $L(G)=D(G)-A(G)$ be the Laplacian matrix of the graph $G$, where $A(G)$ and $D(G)$ are the adjacency matrix and the diagonal matrix of the vertex degrees of $G$, respectively. The normalized Laplacian matrix of $G$ is defined as $L=D(G)^{-\frac{1}{2}} L(G) D(G)^{-\frac{1}{2}}$, where $D(G)^{-\frac{1}{2}}$ is the matrix which is obtained by taking $\left(-\frac{1}{2}\right)$-power of each entry of $D(G)$. The Laplacian eigenvalues and the normalized Laplacian eigenvalues of $G$ are the eigenvalues of $L(G)$ and $L$, respectively. Let $\mu_{1} \geq \mu_{2} \geq \cdots \geq \mu_{n}$ be the Laplacian eigenvalues and $\lambda_{1} \geq \lambda_{2} \geq \cdots \geq \lambda_{n}$ be the normalized Laplacian eigenvalues of $G$. It is well known that $\mu_{n}=0, \lambda_{n}=0$ and the multiplicities of these zero eigenvalues are equal to the number of connected components of $G$; see $[2,3]$.

The number of spanning trees (also known as complexity), $t(G)$, of $G$ is given by the following formula in terms of the Laplacian eigenvalues (see [1], p.39):

$$
t(G)=\frac{1}{n} \prod_{i=1}^{n-1} \mu_{i} .
$$

\section{Springer}

(0) 2012 Bozkurt; licensee Springer. This is an Open Access article distributed under the terms of the Creative Commons Attribution License (http://creativecommons.org/licenses/by/2.0), which permits unrestricted use, distribution, and reproduction in any medium, provided the original work is properly cited. 
It is known that the number of spanning trees of $G$ is also expressed by the normalized Laplacian eigenvalues as follows (see [1], p.49):

$$
t(G)=\left(\frac{\prod_{i=1}^{n} d_{i}}{2 e}\right) \prod_{i=1}^{n-1} \lambda_{i}
$$

Now we list some known upper bounds for $t(G)$.

- Grimmett [4]:

$$
t(G) \leq \frac{1}{n}\left(\frac{2 e}{n-1}\right)^{n-1}
$$

- Grone and Merris [5]:

$$
t(G) \leq\left(\frac{n}{n-1}\right)^{n-1}\left(\frac{\prod_{i=1}^{n} d_{i}}{2 e}\right)
$$

- Nosal [6]: For $r$-regular graphs,

$$
t(G) \leq n^{n-2}\left(\frac{r}{n-1}\right)^{n-1}
$$

- Kelmanns ([1], p.222):

$$
t(G) \leq n^{n-2}\left(1-\frac{2}{n}\right)^{\bar{e}}
$$

where $\bar{e}$ is the number of edges of $\bar{G}$.

- Das [7]:

$$
t(G) \leq\left(\frac{2 e-d_{1}-1}{n-2}\right)^{n-2}
$$

- Zhang [8]:

$$
t(G) \leq(1+(n-2) a)(1-a)^{n-2} \frac{1}{n}\left(\frac{2 e}{n-1}\right)^{n-1}
$$

where $a=\left(\frac{n(n-1)-2 e}{2 e n(n-2)}\right)^{1 / 2}$.

- Feng et al. [9]:

$$
t(G) \leq\left(\frac{d_{1}+1}{n}\right)\left(\frac{2 e-d_{1}-1}{n-2}\right)^{n-2}
$$

and

$$
t(G) \leq\left(\frac{\sum_{i=1}^{n} d_{i}^{2}+2 e-\left(d_{1}+1\right)^{2}}{n-2}\right)^{\frac{n-2}{2}}
$$


- Li et al. [10]:

$$
t(G) \leq d_{n}\left(\frac{2 e-d_{1}-1-d_{n}}{n-3}\right)^{n-3}
$$

In [4] Grimmett observed that (3) is the generalization of (5). Grone and Merris [5] stated that by the application of arithmetic-geometric mean inequality, (4) leads to (3). In [7] Das indicated that (7) is sharp for $S_{n}$ or $K_{n}$, but (3), (4), (5) and (6) are sharp only for $K_{n}$. Li et al. [10] pointed out that (11) is sharp for $S_{n}, K_{n}, G \cong K_{1} \vee\left(K_{1} \cup K_{n-2}\right)$ or $K_{n}-m$, but (3) is sharp only for $K_{n}$, (7) and (9) are sharp for $S_{n}$ or $K_{n}$. In [8,9] the authors showed that (8) is always better than (3), and (9) is always better than (7) and (10).

This paper is organized as follows. In Section 2, we give some useful lemmas. In Section 3, we obtain some upper bounds for the number of spanning trees of graphs in terms of the number of vertices, the number of edges and the vertex degrees of graphs. We also show that one of these upper bounds is always better than the upper bound (4).

\section{Preliminary lemmas}

In this section, we give some lemmas which will be used later. Firstly, we introduce an auxiliary quantity of a graph $G$ on the vertex set $V(G)=\left\{v_{1}, v_{2}, \ldots, v_{n}\right\}$ as

$$
P=1+\sqrt{\frac{2}{n(n-1)} \sum_{v_{i} \sim v_{j}} \frac{1}{d_{i} d_{j}}}
$$

where $d_{i}$ is the degree of the vertex $v_{i}$ of $G$.

Lemma 1 [11] Let $G$ be agraph with $n$ vertices and normalized Laplacian matrix $L$ without isolated vertices. Then

$$
\sum_{i=1}^{n} \lambda_{i}=\operatorname{tr}(L)=n
$$

and

$$
\sum_{i=1}^{n} \lambda_{i}^{2}=\operatorname{tr}\left(L^{2}\right)=n+2 \sum_{v_{i} \sim v_{j}} \frac{1}{d_{i} d_{j}} .
$$

Lemma 2 [3] Let $G$ be a graph with $n$ vertices and normalized Laplacian eigenvalues $\lambda_{1} \geq$ $\lambda_{2} \geq \cdots \geq \lambda_{n}=0$. Then

$$
0 \leq \lambda_{i} \leq 2
$$

Moreover, $\lambda_{1}=2$ if and only if a connected component of $G$ is bipartite and nontrivial.

Lemma 3 [3] Let $G$ be a graph with $n$ vertices and normalized Laplacian eigenvalues $\lambda_{1} \geq$ $\lambda_{2} \geq \cdots \geq \lambda_{n}=0$. Then

$$
\lambda_{1} \geq \frac{n}{n-1} .
$$

Moreover, the equality holds in (12) if and only if $G$ is a complete graph $K_{n}$. 
Lemma 4 [12] Let $G$ be a graph with $n$ vertices and normalized Laplacian eigenvalues $\lambda_{1} \geq \lambda_{2} \geq \cdots \geq \lambda_{n}=0$. Then

$$
\lambda_{1} \geq P .
$$

Moreover, the equality holds in (13) if and only if $G$ is a complete graph $K_{n}$.

Lemma 5 [12] The lower bound (13) is always better than the lower bound (12).

Lemma 6 [12] Let $G$ be a connected graph with $n>2$ vertices. Then $\lambda_{2}=\lambda_{3}=\cdots=\lambda_{n-1}$ if and only if $G \cong K_{n}$ or $G \cong K_{p, q}$.

Lemma 7 [13] Let $G$ be a graph with $n$ vertices and without isolated vertices. Suppose $G$ has the maximum vertex degree equal to $d_{1}$. Then

$$
\sum_{v_{i} \sim v_{j}} \frac{1}{d_{i} d_{j}} \geq \frac{n}{2 d_{1}}
$$

Moreover, the equality holds in (14) if and only if $G$ is a regular graph.

Lemma 8 [14] Let $x_{i}>-1$ for $1 \leq i \leq n$. If $\sum_{i=1}^{n} x_{i}=0$ and $\sum_{i=1}^{n} x_{i}^{2} \geq c^{2}\left(1-n^{-1}\right)$, then

$$
\sum_{i=1}^{n} \ln \left(1+x_{i}\right) \leq \ln \left(1+c-c n^{-1}\right)+(n-1) \ln \left(1-c n^{-1}\right) .
$$

\section{Main results}

Now we present the main results of this paper following the ideas in [8] and [9]. Note that $P$ was defined earlier in the previous section.

Theorem 1 Let $G$ be a graph with $n$ vertices and without isolated vertices. Then

$$
t(G) \leq(1+(n-2) b)(1-b)^{n-2}\left(\frac{n}{n-1}\right)^{n-1}\left(\frac{\prod_{i=1}^{n} d_{i}}{2 e}\right),
$$

where $b=\left(\frac{n-1-d_{1}}{n(n-2) d_{1}}\right)^{1 / 2}$.

Proof If $G$ is disconnected, then $t(G)=0$ and (15) follows. Now we assume that $G$ is connected. From (2), we have

$$
0<t(G)=\left(\frac{\prod_{i=1}^{n} d_{i}}{2 e}\right) \lambda_{1} \cdots \lambda_{n-1}
$$

since $\lambda_{n-1}>0$. Let $q=\frac{n}{n-1}$ and $x_{i}=\frac{\lambda_{i}}{q}-1$ for $1 \leq i \leq n-1$. Then $x_{i}>-1$. Moreover, by Lemma 1 and Lemma 7 , we get

$$
\sum_{i=1}^{n-1} x_{i}=\sum_{i=1}^{n-1}\left(\frac{\lambda_{i}}{q}-1\right)=0
$$


and

$$
\begin{aligned}
\sum_{i=1}^{n-1} x_{i}^{2} & =\sum_{i=1}^{n-1}\left(\frac{\lambda_{i}}{q}-1\right)^{2} \\
& =(n-1)-\frac{2 \sum_{i=1}^{n-1} \lambda_{i}}{q}+\frac{\sum_{i=1}^{n-1} \lambda_{i}^{2}}{q^{2}} \\
& \geq(n-1)-2(n-1)+\left(\frac{n-1}{n}\right)^{2}\left(n+\frac{n}{d_{1}}\right) \\
& =\frac{(n-1)^{2}}{n d_{1}}-\left(\frac{n-1}{n}\right) \\
& =\frac{(n-1)^{2}\left(n-1-d_{1}\right)}{n(n-2) d_{1}}\left(1-\frac{1}{n-1}\right) \\
& =((n-1) b)^{2}\left(1-\frac{1}{n-1}\right) .
\end{aligned}
$$

Then by Lemma 8, we obtain

$$
\prod_{i=1}^{n-1}\left(1+x_{i}\right) \leq\left(1+(n-1) b-\frac{(n-1) b}{n-1}\right)(1-b)^{n-2} .
$$

Therefore, we arrive at

$$
\prod_{i=1}^{n-1} \lambda_{i} \leq(1+(n-2) b)(1-b)^{n-2}\left(\frac{n}{n-1}\right)^{n-1}
$$

and

$$
t(G) \leq(1+(n-2) b)(1-b)^{n-2}\left(\frac{n}{n-1}\right)^{n-1}\left(\frac{\prod_{i=1}^{n} d_{i}}{2 e}\right)
$$

Hence, the result holds.

Remark 1 Let $f(b)=(1+(n-2) b)(1-b)^{n-2}$. Then

$$
f^{\prime}(b)=-(n-2)(n-1) b(1-b)^{n-3} \leq 0
$$

for $0 \leq b \leq 1$. Therefore, $f(b) \leq f(0)=1$; see [8]. Hence, we conclude that the upper bound (15) is always better than the upper bound (4). Moreover, if $G$ is the complete graph $K_{n}$, then the equality holds in (15).

Theorem 2 Let $G$ be a connected graph with $n>2$ vertices. Then

$$
t(G) \leq P\left(\frac{n-P}{n-2}\right)^{n-2}\left(\frac{\prod_{i=1}^{n} d_{i}}{2 e}\right)
$$

Moreover, the equality holds in (16) if and only if $G$ is the complete graph $K_{n}$. 
Proof From (2) and Lemma 1, we get

$$
\begin{aligned}
t(G) & =\left(\frac{\prod_{i=1}^{n} d_{i}}{2 e}\right) \prod_{i=1}^{n-1} \lambda_{i}=\left(\frac{\prod_{i=1}^{n} d_{i}}{2 e}\right) \lambda_{1} \prod_{i=2}^{n-1} \lambda_{i} \\
& \leq\left(\frac{\prod_{i=1}^{n} d_{i}}{2 e}\right) \lambda_{1}\left(\frac{\sum_{i=2}^{n-1} \lambda_{i}}{n-2}\right)^{n-2} \\
& =\left(\frac{\prod_{i=1}^{n} d_{i}}{2 e}\right) \lambda_{1}\left(\frac{\sum_{i=1}^{n-1} \lambda_{i}-\lambda_{1}}{n-2}\right)^{n-2}=\left(\frac{\prod_{i=1}^{n} d_{i}}{2 e}\right) \lambda_{1}\left(\frac{n-\lambda_{1}}{n-2}\right)^{n-2} .
\end{aligned}
$$

For $P \leq x \leq 2$, let

$$
f(x)=x(n-x)^{n-2} .
$$

By Lemma 4 and Lemma 5, we have that

$$
\lambda_{1} \geq P \geq \frac{n}{n-1}
$$

and

$$
f^{\prime}(x)=f(x) \frac{n-(n-1) x}{x(n-x)} \leq 0
$$

for $P \leq x \leq 2$. Hence, $f(x)$ takes its maximum value at $x=P$ and (16) follows.

If the equality holds in (16), then all inequalities in the above argument must be equalities. Hence, we have

$$
\lambda_{1}=P \quad \text { and } \quad \lambda_{2}=\cdots=\lambda_{n-1} .
$$

Then by Lemma 4 and Lemma 6 , we conclude that $G$ is the complete graph $K_{n}$.

Conversely, we can easily see that the equality holds in (16) for the complete graph $K_{n}$.

Now we consider the bipartite graph case of the above theorem.

Theorem 3 Let $G$ be a connected bipartite graph with $n>2$ vertices. Then

$$
t(G) \leq \frac{\prod_{i=1}^{n} d_{i}}{e}
$$

Moreover, the equality holds in (17) if and only if $G \cong K_{p, q}$.

Proof Since $G$ is a connected bipartite graph, by Lemma 2, we have $\lambda_{1}=2$. Considering this, (2) and Lemma 1, we obtain

$$
\begin{aligned}
t(G) & =\left(\frac{\prod_{i=1}^{n} d_{i}}{2 e}\right) \prod_{i=1}^{n-1} \lambda_{i}=\left(\frac{\prod_{i=1}^{n} d_{i}}{2 e}\right) \lambda_{1} \prod_{i=2}^{n-1} \lambda_{i} \\
& \leq\left(\frac{\prod_{i=1}^{n} d_{i}}{e}\right)\left(\frac{\sum_{i=2}^{n-1} \lambda_{i}}{n-2}\right)^{n-2}=\left(\frac{\prod_{i=1}^{n} d_{i}}{e}\right)\left(\frac{n-\lambda_{1}}{n-2}\right)^{n-2}=\frac{\prod_{i=1}^{n} d_{i}}{e} .
\end{aligned}
$$


Moreover, the equality holds in (17) if and only if $\lambda_{2}=\cdots=\lambda_{n-1}$, by Lemma 6, i.e., if and only if $G \cong K_{p, q}$.

\section{Competing interests}

The author declares that she has no competing interests.

\section{Acknowledgements}

The author thanks the referees for their helpful comments and suggestions concerning the presentation of this paper. The author is also thankful to TUBITAK and the Office of Selcuk University Scientific Research Project (BAP). This study is based on a part of the author's PhD thesis.

Received: 15 August 2012 Accepted: 6 November 2012 Published: 22 November 2012

\section{References}

1. Cvetković, D, Doob, M, Sachs, H: Spectra of Graphs. Academic Press, New York (1980)

2. Fiedler, M: Algebraic connectivity of graphs. Czechoslov. Math. J. 23, 298-305 (1973)

3. Chung, FRK: Spectral Graph Theory. Am. Math. Soc., Providence (1997)

4. Grimmett, GR: An upper bound for the number of spanning trees of a graph. Discrete Math. 16, 323-324 (1976)

5. Grone, R, Merris, R: A bound for the complexity of a simple graph. Discrete Math. 69, 97-99 (1988)

6. Nosal, E: Eigenvalues of graphs. Master Thesis, University of Calgary (1970)

7. Das, KC: A sharp upper bound for the number of spanning trees of a graph. Graphs Comb. 23, 625-632 (2007)

8. Zhang, X: A new bound for the complexity of a graph. Util. Math. 67, 201-203 (2005)

9. Feng, L, Yu, G, Jiang, Z, Ren, L: Sharp upper bounds for the number of spanning trees of a graph. Appl. Anal. Discrete Math. 2, 255-259 (2008)

10. Li, J, Shiu, WC, Chang, A: The number of spanning trees of a graph. Appl. Math. Lett. 23, 286-290 (2010)

11. Zumstein, P: Comparison of spectral methods through the adjacency matrix and the Laplacian of a graph. Diploma Thesis, ETH Zürich (2005)

12. Das, KC, Güngör, AD, Bozkurt, ŞB: On the normalized Laplacian eigenvalues of graphs. Ars Comb. (in press)

13. Shi, L: Bounds on Randić indices. Discrete Math. 309, 5238-5241 (2009)

14. Cohn, JHE: Determinants with elements \pm 1 . J. Lond. Math. Soc. 42, 436-442 (1967)

doi:10.1186/1029-242X-2012-269

Cite this article as: Bozkurt: Upper bounds for the number of spanning trees of graphs. Journal of Inequalities and Applications 2012 2012:269.

\section{Submit your manuscript to a SpringerOpen ${ }^{\circ}$ journal and benefit from:}

- Convenient online submission

- Rigorous peer review

- Immediate publication on acceptance

- Open access: articles freely available online

- High visibility within the field

- Retaining the copyright to your article 